\title{
SELF-INTERSECTIONS IN CONTINUOUS RANDOM WALK
}

\author{
BY Z. A. MELZAK ${ }^{1}$
}

Communicated by H. P. McKean, Jr., June 29, 1970

An $n$-step random walk $(n \geqq 3)$ is a sequence of $n$ straight segments, called steps, in the plane; each step is of length 1 , the first step starts at the origin and each successive step starts at the end of the previous one; every step is in random direction with uniform distribution in angle. Neglecting certain events of probability 0 we define a self-intersection as the event when for some $i$ and $j$, with $1 \leqq i<j \leqq n$ and $j-i>1$, the $i$ th and the $j$ th step have in common exactly one point, interior to each step. Let $f(n)$ be the expected number of self-intersections; it is proved that

$$
\begin{array}{r}
f(n)=\frac{n}{4} \sum_{p=2}^{n-1}\left(1-\frac{p}{n}\right) \\
{\left[1-\frac{4}{\pi^{2}} \int_{0}^{\infty} \int_{0}^{\infty}(u v)^{-1} J_{0}(v) \cdot\left[J_{0}^{p-1}(u-v)-J_{0}^{p-1}(u+v)\right] d u d v\right.} \\
+\frac{1}{\pi^{5}} \int_{0}^{\infty} \int_{0}^{\infty} \int_{0}^{\infty} \int_{0}^{\infty} \int_{0}^{2 \pi}(u v w z)^{-1} \sum_{i=1}^{8} \epsilon_{i} \cos \left(c_{i} \sin \theta\right) \\
\left.\cdot J_{0}^{p-1}\left(\left(a_{i}^{2}+2 \eta_{i} a_{i} b_{i} \cos \theta+b_{i}^{2}\right)^{1 / 2}\right) d \theta d u d v d w d z\right]
\end{array}
$$

where $J_{0}$ is the Bessel function of the first kind and zero order, and the quantities indexed by $i$ are as given below:

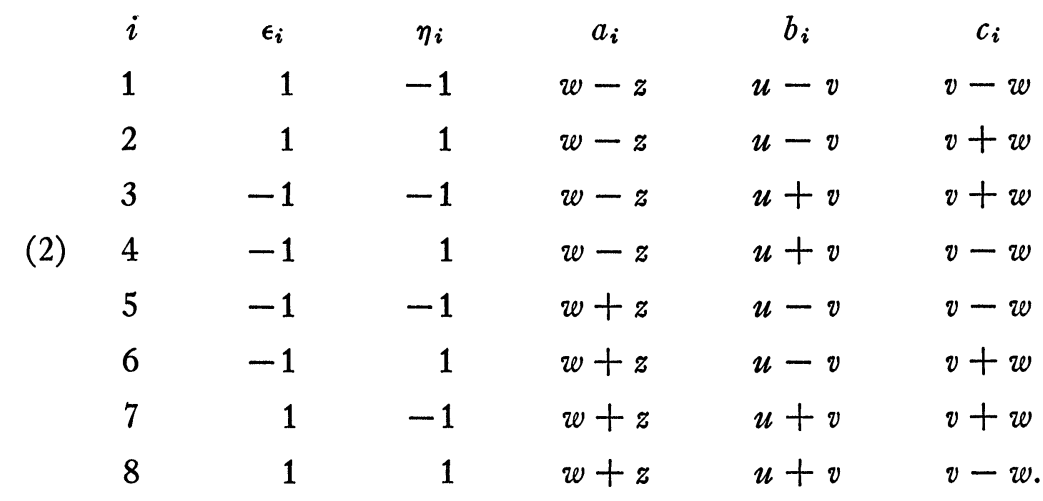

AMS 1970 subject classifications. Primary 6066.

Key words and phrases. Random walk, self-crossings.

${ }^{1}$ Research reported upon was supported by the N. R. C. of Canada. 
It is further proved that for large $n$ we have asymptotically

$$
f(n) \sim\left(2 / \pi^{2}\right) n \log n
$$

where $2 / \pi^{2}$ has a simple probabilistic meaning here: it is four times the probability that a random unit step from a random point inside $x^{2}+y^{2}=4$ intersects the segment $[0,1]$ on the $x$-axis.

An outline of the proof follows. Let the $i$ th step be from $\left(x_{i}, y_{i}\right)$ to $\left(x_{i+1}, y_{i+1}\right)$ and let it make the angle $\alpha_{i}$ with the $x$-axis. The line $L_{i}$ which carries it has the equation

$$
\left(y-y_{i}\right) \cos \alpha_{i}-\left(x-x_{i}\right) \sin \alpha_{i}=0
$$

which we write as $L_{i}(x, y)=0$. For the coordinates $x_{i}$ and $y_{i}$ we have

$$
x_{i}=\sum_{1}^{i-1} \cos \alpha_{k}, \quad y_{i}=\sum_{1}^{i-1} \sin \alpha_{k} .
$$

Since we exclude from consideration the 0 -probability event that any $\left(x_{i}, y_{i}\right)$ lies on $L_{j}$ with $j$ other than $i-1$ or $i$, a self-intersection occurs if and only if

$$
L_{i}\left(x_{j}, y_{j}\right) L_{i}\left(x_{j+1}, y_{j+1}\right)<0 \text { and } L_{j}\left(x_{i}, y_{i}\right) L_{j}\left(x_{i+1}, y_{i+1}\right)<0 .
$$

Hence the total number $F=F\left(\alpha_{1}, \cdots, \alpha_{n}\right)$ of self-intersections is

$$
\begin{array}{r}
F=\frac{1}{4} \sum_{j=3}^{n} \sum_{i=1}^{j-2}\left[1-\operatorname{sgn} L_{i}\left(x_{j}, y_{j}\right) \operatorname{sgn} L_{i}\left(x_{j+1}, y_{j+1}\right)\right] \\
\cdot\left[1-\operatorname{sgn} L_{j}\left(x_{i}, y_{i}\right) \operatorname{sgn} L_{j}\left(x_{i+1}, y_{i+1}\right)\right]
\end{array}
$$

where to represent the discontinuous factor $\operatorname{sgn} x$ we use

$$
\operatorname{sgn} x=\frac{2}{\pi} \int_{0}^{\infty} u^{-1} \sin u x d u .
$$

The expected number $f(n)$ of self-intersections is

$$
f(n)=(2 \pi)^{-n} \int_{0}^{2 \pi} \cdots \int_{0}^{2 \pi} F d \alpha_{1} \cdots d \alpha_{n},
$$

the above form of $F$ is substituted into the $n$-tuple integral, the order of integrations is changed, and the angle integrations are carried out, leading after some lengthy transformations to (1). For the proof of (3) we use the standard asymptotics of the Bessel functions, representing $J_{0}^{p}(x)$ as $\exp \left(-p x^{2} / 4\right)$ times a power series.

It is expected that the detailed proofs, the numerical work, and further related material will appear elsewhere.

The University of British Columbia, Vancouver, Canada 